\title{
Global existence for the two-dimensional Kuramoto-Sivashinsky equation with a shear flow
}

\author{
Michele Coti Zelati, Michele Dolce, Yuanyuan Feng and \\ ANNA L. MAZZUCATO
}

Abstract. We consider the Kuramoto-Sivashinsky equation (KSE) on the two-dimensional torus in the presence of advection by a given background shear flow. Under the assumption that the shear has a finite number of critical points and there are linearly growing modes only in the direction of the shear, we prove global existence of solutions with data in $L^{2}$, using a bootstrap argument. The initial data can be taken arbitrarily large.

\section{Introduction}

In this article we consider the Kuramoto-Sivashinsky equation (KSE) in two-space dimension in the presence of advection by a given background shear flow. The KSE is a well-known model of large-scale instabilities, such as those arising in flame-front propagation (see e.g. [26] and references therein).

The KSE comes in a scalar, potential form, and a differentiated, vectorial form. We will confine ourselves to the scalar form, since the addition of a linear transport term is meaningful for the potential:

$$
\partial_{t} \phi+\frac{1}{2}|\nabla \phi|^{2}+\Delta^{2} \phi+\Delta \phi=0 .
$$

We solve this equation with periodic boundary conditions on $\left[0, L_{1}\right] \times\left[0, L_{2}\right]$, that is, on a two-dimensional torus, which with slight abuse of notation we denote by $\mathbb{T}^{2}$. When $L_{1}>2 \pi$ or $L_{2}>2 \pi$, the symbol of the linear operator $\Delta^{2}+\Delta$ is negative

Mathematics Subject Classification: 35K25, 35K58, 76E06, 76F25

Keywords: Two dimension, Kuramoto-Sivashinsky, Mixing, Global existence, Mild solutions, Enhanced diffusion, Diffusion time.

On behalf of all authors, the corresponding author states that there is no conflict of interest. Data sharing not applicable to this article as no datasets were generated or analyzed during the current study. A.M. was partially supported by the US National Science Foundation Grant DMS-1909103. Part of this work was conducted while A.M. participated remotely in a program hosted by the Mathematical Sciences Research Institute (MSRI) in Berkeley, California, during the Spring 2021 semester. MSRI receives major support from the US National Science Foundation under Grant DMS-1928930. M.C.Z. and M.D. acknowledges funding from the Royal Society through a University Research Fellowship (URF/R1/191492). 
on a finite set of low frequencies. Hence, there are (linearly) growing modes in the horizontal or vertical direction, respectively.

Since the Kuramoto-Sivashinsky equation describe the motion of a (plane) frame front $[31,37]$, one and two space dimensions are the most physically relevant for the problem. For the same reason, taking periodic boundary conditions is physically motivated, even though other geometries, such as that of a channel, are of interest. We confine ourselves to treating periodic boundary conditions.

We consider a modified version of (1), where the potential $\phi$ is subject to advection by a given steady shear flow, which we write without loss of generality as the horizontal shear $\mathbf{v}=(u(y), 0)$ :

$$
\partial_{t} \phi+A u(y) \partial_{x} \phi+\frac{1}{2}|\nabla \phi|^{2}+\Delta^{2} \phi+\Delta \phi=0,
$$

where the parameter $A>0$ represents the amplitude of the flow. As we explain more precisely below, we are interested in considering shear flows which are relaxation enhancing [11], for instance, a sufficient condition [12] is that $u$ has a finite number of critical points. The KSE with general advection term has been utilized in models of turbulent premixed-combustion [15]. By a change of time, the above equation can be rewritten in an equivalent way as

$$
\partial_{t} \phi+u(y) \partial_{x} \phi+\frac{v}{2}|\nabla \phi|^{2}+v \Delta^{2} \phi+v \Delta \phi=0
$$

where $v=A^{-1}$ and, with slight abuse of notation, we have not relabeled the transformed variables. Because $v$ determines the strength of the dissipation, we will refer to $v$ as a viscosity coefficient. We will refer to the equation above as AKSE.

The main difficulty in dealing with both (1) and (2) is the lack of a priori norm estimates on the solution, which does not allow to bootstrap local existence into global existence via a standard continuation argument. The analysis of the KSE in one space dimension is well developed by now, since in one dimension energy estimates lead to a good control on the $L^{2}$ norm of the solution [8,9,21-24,35]. By contrast, there are only a handful of results concerning the well-posedness of the classical KSE (1) in dimension greater than one. Local well-posedness holds in $L^{p}$ spaces [7,27]. Global existence is known only under fairly restrictive assumptions, such as for thin domains and for the anisotropically reduced $\operatorname{KSE}[6,30,32,36]$, without growing modes $[1,17]$, or with only one growing mode in each direction [2], for small data. The attractor and determining modes were studied in [34], under a uniform bound on higher Sobolev norms that yields global existence of solutions.

In [17], two of the authors proved global existence for AKSE for large data and any number of growing modes, when the advecting velocity field induces a sufficiently small dissipation time, e.g. if the flow is mixing, that leads to a global uniform bound on the $L^{2}$ norm of the solution. In this case, the action of the flow is to move energy from large scales to small scales in both directions, where the dissipation can efficiently damp the effect of all the growing modes. We prove in this work that the same result, 
global existence for large data, holds, if the advecting flow is a shear flow with only isolated critical points and in the absence of growing modes in the direction transverse to the shear, the vertical direction in our set up, which can be achieved by restricting $0<L_{2}<2 \pi$. The idea of the proof is to exploit the enhanced dissipation arising from the combined action of the hyper-diffusion and the advection to control both the nonlinearity as well as the destabilizing effect of the negative Laplacean at large scale. Intuitively speaking, the shear flow has no influence on purely vertical modes. For instance, the function of $y$ obtained by averaging the solution in the $x$ direction may grow in time. On the other hand, the mixing along streamlines of the flow moves energy from large to small scales. Therefore, the growth generated by growing horizontal modes is damped on a sufficiently large time-scale by the dissipation. The nonlinearity then couples all the modes.

For the case at hand of a steady shear flow, the transport operator has a large kernel, namely all the functions on the torus that are constant in the horizontal variable. One needs to project out the kernel to take advantage of the action of the flow. There is no enhanced decay of the energy on the kernel component (at a linear level), but the norm can nevertheless be controlled as they satisfy collectively a modified one-dimensional KSE. A key point is to use the fact that the linear operator

$$
H_{v}:=v \Delta^{2}+u(y) \partial_{x},
$$

is dissipation enhancing $[11,12,16]$. More precisely, for the components of the solution orthogonal to the kernel of the transport operator, it generates an exponentially stable semigroup $\mathrm{e}^{-t H_{v}}$ with a rate of decay of the $L^{2}$ norm of order $\lambda_{\nu}$, where $v / \lambda_{v} \rightarrow 0$ as $v \rightarrow 0$. By contrast, a standard energy estimate shows that the semigroup is contractive with rate $O(v)$. The improved rate in viscosity allows to control both the growing modes as well as the nonlinear terms, provided $v$ is small enough compared to the size of the initial data. Given $g \in L^{2}\left(\mathbb{T}^{2}\right)$, we denote

$$
\langle g\rangle(y)=\frac{1}{L_{1}} \int_{\mathbb{T}^{1}} g(t, x, y) \mathrm{d} x, \quad g_{\neq}(x, y)=g(x, y)-\langle g\rangle(y) .
$$

By Fubini-Tonelli's Theorem, $\langle g\rangle$ exists for a.e. $y$. We observe that $\langle g\rangle$ corresponds to the projection of $g$ onto the kernel of the advection operator $u(y) \partial_{x}$, while $g_{\neq}$ corresponds to the projection onto the orthogonal complement in $L^{2}$.

We define the order of a critical point $x_{0}$ of a function $u(x)$ as the smallest $m \in \mathbb{N}$ such that the derivative $u^{m}\left(x_{0}\right) \neq 0$. A simple critical point has therefore order $m=2$.

As shown in [4], if $u$ has a finite number of critical points of order at most $m$, then $u$ is mixing in the sense that for some constant $C>0$ there holds

$$
\left\|\mathrm{e}^{-u \partial_{x} t} g_{\neq}\right\|_{H^{-1}} \leq \frac{C}{(1+t)^{1 / m}}\left\|g_{\neq}\right\|_{H^{1}},
$$

for every $t \geq 0$. Thanks to, [12, Corollary 2.3] this translates into the enhanced dissipation estimate

$$
\left\|\mathrm{e}^{-H_{v} t} g_{\neq}\right\|_{L^{2}} \leq 5 \mathrm{e}^{-\lambda_{v} t}\left\|g_{\neq}\right\|_{L^{2}}, \quad \lambda_{v}=\varepsilon_{0} v^{\frac{2 m}{2 m+1}},
$$


for some $\varepsilon_{0}>0$, independent of $v$, and for every $t \geq 0$. For AKSE, we use (6) to show that solutions are global, as stated in the next theorem.

Theorem 1.1. Let $0<L_{2}<2 \pi$ and let $u:\left[0, L_{2}\right) \rightarrow \mathbb{R}$ be a smooth function with a finite number of critical points of order at most $m$. Then, given $\phi_{0} \in L^{2}\left(\mathbb{T}^{2}\right)$, there exists $0<v_{0}<1$ depending on $L_{1}, L_{2}, u$, and $\left\|\phi_{0}\right\|_{L^{2}}$ with the following property: for any $0<v<v_{0}$, there exists a unique global-in-time weak solution $\phi$ of (2) with initial data $\phi_{0}$ such that $\phi \in L^{\infty}\left([0, \infty), L^{2}\right) \cap L^{2}\left([0, \infty), H^{2}\right) 0<T<\infty$

We stress that we can allow any number of growing modes in the horizontal direction and the initial data can be arbitrarily large in $L^{2}$. The uniqueness of solutions in $C\left([0, T], L^{2}\right)$ follows similarly to other semi-linear parabolic equations (see e.g. [38, Proposition 1.1, page 315]).

The proof is based on a bootstrap argument inspired by [5]. The main steps in this argument are as follows. For any initial data in $L^{2}$, there exists a local-in-time mild solution of (2) on some interval of time $\left[0, t_{0}\right)$, which is also a weak solution in $L^{\infty}\left(\left[0, t_{0}\right), L^{2}\right) \cap L^{2}\left(\left[0, t_{0}\right), H^{2}\right)$ and satisfies the energy identity [17]. For $t_{0}$ small enough, we can make the $L^{2}$ and $H^{2}$ norms of the projected component less than a certain multiple of the size of the initial data. By using the stability of the semigroup generated by $H_{v}$, one then shows that, for $v$ sufficiently small, these norms are in fact half that amount. Hence, the solution can be continued for a longer time than $t_{0}$, which allows to bootstrap existence from local to global for the projected component and then conclude using the time evolution of the kernel component of the solution.

As we shall see in Sect. 2, the size of $v_{0}$ in Theorem 1.1 depends on the rate at which $v / \lambda_{v}$ vanishes as $v \rightarrow 0$. Hence, improving the semigroup estimate (6) automatically implies a better global existence threshold. In Sect. 3, we show that imposing a possibly more restrictive condition on $u$ (see Assumption 3.1), the semigroup bound can be improved. In particular, we consider as a prototypical example the case of

$$
u(y)=\sin \left((2 \pi y) / L_{2}\right)^{\ell} \quad \text { for } \ell \in \mathbb{N},
$$

and prove the following result.

Proposition 1.2. Let $g \in L^{2}\left(\mathbb{T}^{2}\right), 0<v<1$, and $u(y)$ be given as in (7). There exists $\varepsilon_{0}^{\prime}>0$, independent of $v$, such that

$$
\left\|\mathrm{e}^{-t H_{v}} g_{\neq}\right\|_{L^{2}} \leq \mathrm{e}^{-\lambda_{v}^{\prime} t+\pi / 2}\left\|g_{\neq}\right\|_{L^{2}}, \quad \lambda_{v}^{\prime}=\varepsilon_{0}^{\prime} v^{\frac{\max \{2, \ell\}}{\max \{2, \ell\}+4}},
$$

for every $t \geq 0$.

We observe that $\ell$ is related to $m$ in (6) in the sense that $u$ in (7) has critical points of order at most $m=\max \{2, \ell\}$. Hence, a direct comparison between (6) and (8) shows that (8) has a much better decay rate, and in particular $v / \lambda_{v}^{\prime} \rightarrow 0$ faster as $v \rightarrow 0$.

The derivation of the semigroup estimate (8) is carried out in Sect. 3 via a spectraltheoretic approach. It follows from a general Gearhart-Prüss criterion for m-accretive operators devised in [39] based on a quantitative pseudo-spectral bound. The proof is 
motivated by that of a similar result for the Laplace operator $\Delta$ in [20]. For the Laplace operator plus advection, decay rates akin to (8) were obtained in [10,39] for a shear with infinitely many critical points, using the pseudo-spectral approach, and for shear flows with finitely many critical points in [4], using hypocoercivity. Such quantitative semigroup estimates are relevant in the investigation of enhanced diffusion for passive scalars $[3,4,13,39]$, in the study of asymptotic stability of particular solutions to the two-dimensional Navier-Stokes equations [14,19,33,40], and have also applications to several other nonlinear problems [5,25,28,29].

In Sect. 3, we prove a more general version of Proposition 1.2, namely Proposition 1.1, for shear flows satisfying a certain condition, Assumption 3.1, again inspired by [20]. This condition can be readily verified for $u$ in (7). This is a main reason while we chose it as prototypical example. In fact, by refining the method of proof, we expect an analog of Proposition 1.2 to hold for any shear flow with critical points of order $m$.

In what follows, $C$ denotes a generic constant that may depend on the domain, i.e., on $L_{1}$ and $L_{2}$. We utilize standard notation to denote function spaces, e.g. $H^{k}\left(\mathbb{T}^{2}\right)$ is the usual $L^{2}$-based Sobolev space. We denote the $L^{2}$-norm on the whole $\mathbb{T}^{2}$ as $\|\cdot\|_{L^{2}}$ while $\|\cdot\|_{L_{y}^{2}}$ is the $L^{2}$-norm on the torus in the vertical direction.

Finally, the paper is organized as follows. In Sect. 2, we obtain the bootstrap estimates and prove Theorem 1.1. Then, in Sect. 3, we establish the exponential stability of the semigroup generated by $H_{v}$ with the improved decay rate, using spectral estimates.

\section{Global existence for the KSE with shear}

In this section, we establish global existence of solutions of the KSE in the presence of advection by a shear flow with a finite number of critical points. The semigroup estimate (6) allows to control these growing modes through a suitable decomposition of the solution and a bootstrap argument.

\subsection{Decomposition of the solution and proof of the main result}

In this section, we derive the system of coupled equations that describe the time evolution of the component $\langle\phi\rangle$ of the solution in the kernel of the transport operator and the time evolution of the component $\phi_{\neq}$in the orthogonal complement.

We will refer informally to $\langle\phi\rangle$ and $\phi_{\neq}$as the kernel and projected components, respectively. Then $\langle\phi\rangle$ satisfies

$$
\partial_{t}\langle\phi\rangle+\frac{v}{2 L_{1}} \int_{\mathbb{T}^{1}}\left|\nabla \phi_{\neq}+\nabla\langle\phi\rangle\right|^{2} \mathrm{~d} x+v \partial_{y}^{4}\langle\phi\rangle+v \partial_{y}^{2}\langle\phi\rangle=0,
$$


while $\phi_{\neq}$satisfies

$$
\begin{aligned}
& \partial_{t} \phi_{\neq}+u(y) \partial_{x} \phi_{\neq}+v \Delta^{2} \phi_{\neq} \\
& =-\frac{v}{2}\left|\nabla \phi_{\neq}+\nabla\langle\phi\rangle\right|^{2}+\frac{v}{2 L_{1}} \int_{\mathbb{T}^{1}}\left|\nabla \phi_{\neq}+\nabla\langle\phi\rangle\right|^{2} \mathrm{~d} x-v \Delta \phi_{\neq} \\
& =-\frac{v}{2}\left|\nabla \phi_{\neq}\right|^{2}+\frac{v}{2}\left\langle\left|\nabla \phi_{\neq}\right|^{2}\right\rangle-v \partial_{y} \phi_{\neq} \partial_{y}\langle\phi\rangle-v \Delta \phi_{\neq} .
\end{aligned}
$$

We remark that in the equation above the kernel component interacts with the projected ones through the term $\partial_{y}\langle\phi\rangle$. Denoting $\psi=\partial_{y}\langle\phi\rangle$ for notational ease, we have

$$
\partial_{t} \psi+\frac{v}{2 L_{1}} \int_{\mathbb{T}^{1}} \partial_{y}\left|\nabla \phi_{\neq}\right|^{2} \mathrm{~d} x+v \psi \partial_{y} \psi+v \partial_{y}^{4} \psi+v \partial_{y}^{2} \psi=0 .
$$

It was proved in [17] that the unique local mild solution to (2) is also a weak solution satisfying the energy identity on the time of existence of the mild solution. In particular, $\phi_{\neq} \in L^{\infty}\left(\left(0, t_{0}\right) ; L^{2}\left(\mathbb{T}^{2}\right)\right) \cap L^{2}\left(\left(0, t_{0}\right) ; H^{2}\left(\mathbb{T}^{2}\right)\right)$, at least for a sufficiently small time $t_{0}>0$. Furthermore, it was shown in [17] that the mild and weak solution persists as long as its $L^{2}$ norm is finite, that is, if $T^{*}$ is the maximal time of existence of the solution, then

$$
T^{*}<\infty \Rightarrow \limsup _{t \rightarrow T^{*}}\|\phi(t)\|_{L^{2}}=\infty
$$

Our goal is to obtain a global bound on the $L^{2}$ norm of the solution via a bootstrap argument, from which global existence follows. We will employ both energy estimates as well as semigroup estimates to exploit enhanced dissipation arising from the addition of the advection term on $\phi_{\neq}$.

Let $\mathcal{S}_{t}$ be the solution operator from 0 to time $t \geq 0$ for the transport-hyperdiffusion equation:

$$
\partial_{t} g+u(y) \partial_{x} g+v \Delta^{2} g=0
$$

that is, $\mathcal{S}_{t}=\mathrm{e}^{-t H_{\nu}}$. Then $\phi_{\neq}$satisfies for $0 \leq \bar{t} \leq t$,

$$
\begin{aligned}
\phi_{\neq}(t)= & \mathcal{S}_{t-\bar{t}}\left(\phi_{\neq}(\bar{t})\right)+ \\
& +\int_{0}^{t-\bar{t}} \mathcal{S}_{t-\bar{t}-s}\left(-\frac{v}{2}\left|\nabla \phi_{\neq}(s+\bar{t})\right|^{2}\right. \\
& \left.+\frac{v}{2}\left\langle\left|\nabla \phi_{\neq}(s+\bar{t})\right|^{2}\right\rangle-v \psi(s) \partial_{y} \phi_{\neq}(s+\bar{t})-v \Delta \phi_{\neq}(s+\bar{t})\right) \mathrm{d} s,
\end{aligned}
$$

by Duhamel's principle. We note that the "forcing" term under the integral sign on the right-hand side of this equation is well controlled as long as $\phi_{\neq} \in L^{\infty}\left(\left(0, t_{0}\right) ; L^{2}\left(\mathbb{T}^{2}\right)\right) \cap$ $L^{2}\left(\left(0, t_{0}\right) ; H^{2}\left(\mathbb{T}^{2}\right)\right)$, provided $\psi$ is also controlled. 
Using the decay of $\mathcal{S}_{t}$ on the projected component given by (6), it follows from (12) that, for $0 \leq s \leq t$,

$$
\begin{aligned}
\left\|\phi_{\neq}(t)\right\|_{L^{2}} \leq & \left\|\mathcal{S}_{t}\left(\phi_{\neq}(s)\right)\right\|_{L^{2}}+C v \int_{0}^{t-s}\left(\left\|\nabla \phi_{\neq}\right\|_{L^{4}}^{2}\right. \\
& \left.+\|\psi\|_{L_{y}^{4}}\left\|\nabla \phi_{\neq}\right\|_{L^{4}}+\left\|\Delta \phi_{\neq}\right\|_{L^{2}}\right)(s+\tau) \mathrm{d} \tau \\
\leq & \left\|\mathcal{S}_{t}\left(\phi_{\neq}(s)\right)\right\|_{L^{2}}+C v \int_{0}^{t-s}\left(\left\|\phi_{\neq}\right\|_{L^{2}}^{1 / 2}\left\|\Delta \phi_{\neq}\right\|_{L^{2}}^{3 / 2}+\left\|\Delta \phi_{\neq}\right\|_{L^{2}}\right. \\
& \left.+\left\|\phi_{\neq}\right\|_{L^{2}}^{1 / 4}\left\|\Delta \phi_{\neq}\right\|_{L^{2}}^{3 / 4}\|\psi\|_{L_{y}^{2}}^{7 / 8}\left\|\partial_{y}^{2} \psi\right\|_{L_{y}^{2}}^{1 / 8}\right)(s+\tau) \mathrm{d} \tau \\
\leq & \left\|\mathcal{S}_{t}\left(\phi_{\neq}(0)\right)\right\|_{L^{2}}+C v \int_{0}^{t}\left(\left\|\phi_{\neq}\right\|_{L^{2}}^{1 / 2}\left\|\Delta \phi_{\neq}\right\|_{L^{2}}^{3 / 2}\right. \\
& \left.+\left\|\Delta \phi_{\neq}\right\|\left\|_{L^{2}}+\right\| \phi_{\neq}\left\|_{L^{2}}^{1 / 4}\right\| \Delta \phi_{\neq}\left\|_{L^{2}}^{3 / 4}\right\| \partial_{y}^{2} \psi \|_{L_{y}^{2}}\right)(s+\tau) \mathrm{d} s,
\end{aligned}
$$

where in the above estimate we used the fact $\|\psi\|_{L_{y}^{2}} \leq C\left\|\partial_{y}^{2} \psi\right\|_{L_{y}^{2}}$ by applying Poincaré's inequality twice (we exploit here that $\psi$ and, hence, all its derivatives have zero average by definition), and the following Gagliardo-Nirenberg interpolation inequalities:

$$
\left\|\nabla \phi_{\neq}\right\|_{L^{4}} \leq C\left\|\phi_{\neq}\right\|_{L^{2}}^{1 / 4}\left\|\Delta \phi_{\neq}\right\|_{L^{2}}^{3 / 4}, \quad\|\psi\|_{L_{y}^{4}} \leq C\|\psi\|_{L_{y}^{2}}^{7 / 8}\left\|\partial_{y}^{2} \psi\right\|_{L_{y}^{2}}^{1 / 8} .
$$

We next derive some energy estimates that will be needed for the bootstrap argument. Multiplying (10) by $\phi_{\neq}$and integrating by part, using the periodic boundary conditions, yields:

$$
\begin{aligned}
\frac{1}{2} \frac{\mathrm{d}}{\mathrm{d} t}\left\|\phi_{\neq}\right\|_{L^{2}}^{2}+v\left\|\Delta \phi_{\neq}\right\|_{L^{2}}^{2} & -\frac{v}{2} \int_{\mathbb{T}^{2}}\left|\nabla \phi_{\neq}\right|^{2} \phi_{\neq} \mathrm{d} x \mathrm{~d} y+\frac{v}{2 L_{1}} \int_{\mathbb{T}^{2}}\left(\int_{\mathbb{T}^{1}}\left|\nabla \phi_{\neq}\right|^{2} \mathrm{~d} x\right) \phi_{\neq} \mathrm{d} x \mathrm{~d} y \\
& \quad-v \int_{\mathbb{T}^{2}} \psi \partial_{y} \phi_{\neq} \phi_{\neq} \mathrm{d} x \mathrm{~d} y+v\left\|\nabla \phi_{\neq}\right\|_{L^{2}}^{2} \\
\leq & C v\left\|\nabla \phi_{\neq}\right\|_{L^{4}}^{2}\left\|\phi_{\neq}\right\|_{L^{2}}+C v\|\psi\|_{L_{y}^{2}}\left\|\nabla \phi_{\neq}\right\|_{L^{4}}\left\|\phi_{\neq}\right\|_{L^{4}}+v\left\|\nabla \phi_{\neq}\right\|_{L^{2}}^{2}
\end{aligned}
$$

We recall the Gagliardo-Nirenberg interpolation inequalities in (14) and

$$
\left\|\phi_{\neq}\right\|_{L^{4}} \leq C\left\|\phi_{\neq}\right\|_{L^{2}}^{3 / 4}\left\|\Delta \phi_{\neq}\right\|_{L^{2}}^{1 / 4}
$$

These estimate imply:

$$
\begin{aligned}
\frac{1}{2} \frac{\mathrm{d}}{\mathrm{d} t}\left\|\phi_{\neq}\right\|_{L^{2}}^{2}+v\left\|\Delta \phi_{\neq}\right\|_{L^{2}}^{2} \leq & C v\left\|\phi_{\neq}\right\|_{L^{2}}^{3 / 2}\left\|\Delta \phi_{\neq}\right\|_{L^{2}}^{3 / 2}+C v\|\psi\|_{L_{y}^{2}}\left\|\phi_{\neq}\right\|_{L^{2}}\left\|\Delta \phi_{\neq}\right\|_{L^{2}} \\
& +v\left\|\phi_{\neq}\right\|_{L^{2}}\left\|\Delta \phi_{\neq}\right\|_{L^{2}}
\end{aligned}
$$


where we have integrated by parts in the last term in (15). Applying Young's inequality, we further get

$$
\frac{\mathrm{d}}{\mathrm{d} t}\left\|\phi_{\neq}\right\|_{L^{2}}^{2}+v\left\|\Delta \phi_{\neq}\right\|_{L^{2}}^{2} \leq C v\left\|\phi_{\neq}\right\|_{L^{2}}^{6}+C v\left\|\phi_{\neq}\right\|_{L^{2}}^{2}+C v\left\|\phi_{\neq}\right\|_{L^{2}}^{2}\|\psi\|_{L_{y}^{2}}^{2} .
$$

We also recall that the enhanced diffusion estimate (6) for $S_{t}$ :

$$
\left\|\mathcal{S}_{t} g\right\|_{L^{2}} \leq 5 \mathrm{e}^{-\lambda_{v} t}\|g\|_{L^{2}}
$$

for any $g \in L^{2}\left(\mathbb{T}^{2}\right)$ with $\int_{\mathbb{T}^{1}} g(x, y) \mathrm{d} x=0$. Above $\lambda_{\nu}$ satisfies

$$
\frac{v}{\lambda_{v}} \rightarrow 0, \quad \text { as } \quad v \rightarrow 0
$$

In view of (12), the regularity of the mild and weak solution and the continuation principle, for all sufficiently small times $t \geq s \geq 0$ we can assume that (H1) $\left\|\phi_{\neq}(t)\right\|_{L^{2}} \leq 8 \mathrm{e}^{-\lambda_{v}(t-s) / 4}\left\|\phi_{\neq}(s)\right\|_{L^{2}}$, (H2) $v \int_{s}^{t}\left\|\Delta \phi_{\neq}(\tau)\right\|_{L^{2}}^{2} \mathrm{~d} \tau \leq 4\left\|\phi_{\neq}(s)\right\|_{L^{2}}^{2}$.

Let $t_{0}>0$ be the maximal time such that the estimates above hold on $\left[0, t_{0}\right]$. Following [7], we refer to (H1)-(H2) with $t \in\left[0, t_{0}\right]$ as the bootstrap assumptions. The next lemma ensures suitable bounds on $\psi$ once the bootstrap assumptions (H1) and (H2) hold.

Lemma 2.1. Let $0<L_{2}<2 \pi$. Assume the bootstrap assumptions (H1) and (H2). There exists a $v$-independent constant $C_{1}=C_{1}\left(\left\|\phi_{\neq}(0)\right\|_{L^{2}},\|\psi(0)\|_{L_{y}^{2}}\right)$, which can be explicitly computed, such that

$$
\|\psi(t)\|_{L_{y}^{2}}^{2}+v \int_{0}^{t}\left\|\partial_{y}^{2} \psi(s)\right\|_{L_{y}^{2}}^{2} \mathrm{~d} s \leq C_{1},
$$

for all $t \in\left[0, t_{0}\right]$.

Proof. First from the energy estimate and Poincaré's inequality, we have

$$
\begin{aligned}
& \frac{1}{2} \frac{\mathrm{d}}{\mathrm{d} t}\|\psi\|_{L_{y}^{2}}^{2}+v\left\|\partial_{y}^{2} \psi\right\|_{L_{y}^{2}}^{2} \\
& =v\left\|\partial_{y} \psi\right\|_{L_{y}^{2}}^{2}+\frac{v}{2 L_{1}} \int_{\mathbb{T}^{2}}\left|\nabla \phi_{\neq}\right|^{2} \partial_{y} \psi \mathrm{d} x \mathrm{~d} y \\
& \leq v\left(\frac{L_{2}}{2 \pi}\right)^{2}\left\|\partial_{y}^{2} \psi\right\|_{L_{y}^{2}}^{2}+\frac{v}{2 L_{1}^{\frac{1}{2}}}\left\|\nabla \phi_{\neq}\right\|_{L^{4}}^{2}\left\|\partial_{y} \psi\right\|_{L_{y}^{2}} .
\end{aligned}
$$

It follows from the Gagliardo-Nirenberg inequality that

$$
\left\|\partial_{y} \psi\right\|_{L_{y}^{2}}^{2} \leq\left\|\partial_{y}^{2} \psi\right\|_{L_{y}^{2}}\|\psi\|_{L_{y}^{2}} .
$$


Appealing to (14) and the bound above, estimate (23) becomes

$\frac{1}{2} \frac{\mathrm{d}}{\mathrm{d} t}\|\psi\|_{L_{y}^{2}}^{2}+v\left(1-\left(\frac{L_{2}}{2 \pi}\right)^{2}\right)\left\|\partial_{y}^{2} \psi\right\|_{L_{y}^{2}}^{2} \leq C v\left\|\phi_{\neq}\right\|_{L^{2}}^{1 / 2}\left\|\Delta \phi_{\neq}\right\|_{L^{2}}^{3 / 2}\left\|\partial_{y}^{2} \psi\right\|_{L_{y}^{2}}^{1 / 2}\|\psi\|_{L_{y}^{2}}^{1 / 2}$.

We now exploit in a crucial way the hypothesis $L_{2}<2 \pi$. By Young's inequality we deduce that

$$
\begin{aligned}
& \frac{\mathrm{d}}{\mathrm{d} t}\|\psi\|_{L_{y}^{2}}^{2}+v\left(1-\left(\frac{L_{2}}{2 \pi}\right)^{2}\right)\left\|\partial_{y}^{2} \psi\right\|_{L_{y}^{2}}^{2} \leq C v\left\|\phi_{\neq}\right\|_{L^{2}}^{2 / 3}\left\|\Delta \phi_{\neq}\right\|_{L^{2}}^{2}\|\psi\|_{L_{y}^{2}}^{2 / 3} \\
& \leq C v\left\|\phi_{\neq}\right\|_{L^{2}}^{2 / 3}\left\|\Delta \phi_{\neq}\right\|_{L^{2}}^{2}+C v\left\|\phi_{\neq}\right\|_{L^{2}}^{2 / 3}\left\|\Delta \phi_{\neq}\right\|_{L^{2}}^{2}\|\psi\|_{L_{y}^{2}}^{2} .
\end{aligned}
$$

We define an integrating factor $\mu=\exp \left(-C v \int_{0}^{t}\left\|\phi_{\neq}\right\|_{L^{2}}^{2 / 3}\left\|\Delta \phi_{\neq}\right\|_{L^{2}}^{2} \mathrm{~d} s\right)$. Then solving (26) gives

$$
\begin{aligned}
\|\psi(t)\|_{L_{y}^{2}}^{2} & \leq C v \mu^{-1} \int_{0}^{t}\left\|\phi_{\neq}(s)\right\|_{L^{2}}^{2 / 3}\left\|\Delta \phi_{\neq}(s)\right\|_{L^{2}}^{2} \mathrm{~d} s+\mu^{-1}\|\psi(0)\|_{L_{y}^{2}}^{2} \\
& \leq\left(16 C \mathrm{e}^{16 C\left\|\phi_{\neq}(0)\right\|_{L^{2}}^{8 / 3}}\left\|\phi_{\neq}(0)\right\|_{L^{2}}^{8 / 3}+\mathrm{e}^{16 C\left\|\phi_{\neq}(0)\right\|_{L^{2}}^{8 / 3}}\|\psi(0)\|_{L_{y}^{2}}^{2}\right)=: \widetilde{C}_{1},
\end{aligned}
$$

where the last inequality follows by the bootstrap assumptions (H1) and (H2). By using (27) in (25), we get (22).

We show below in Sect. 2.2 that, in fact, there exists $v_{0}>0$ small enough such that, if $v<v_{0}$ and (H1)-(H2) hold on $\left[0, t_{0}\right]$, then for any $0 \leq s \leq t \leq t_{0}$

(B1) $\left\|\phi_{\neq}(t)\right\|_{L^{2}} \leq 4 \mathrm{e}^{-\lambda_{v}(t-s) / 4}\left\|\phi_{\neq}(s)\right\|_{L^{2}}$,

(B2) $v \int_{s}^{t}\left\|\Delta \phi_{\neq}(\tau)\right\|_{L^{2}}^{2} \mathrm{~d} \tau \leq 2\left\|\phi_{\neq}(s)\right\|_{L^{2}}^{2}$.

We refer to (B1)-(B2) as the bootstrap estimates. Thus, under the assumptions (H1)(H2), the stronger conclusions (B1)-(B2) hold. As a consequence, (B1)-(B2) hold for all positive times. Assuming temporarily this fact, we proceed with the proof of Theorem 1.1.

Proof of Theorem 1.1. From Lemmas 2.2 and 2.5 and the definition of $t_{0}$, we must have $t_{0}=\infty$. In particular, $\phi_{\neq} \in L^{\infty}\left([0, \infty) ; L^{2}\left(\mathbb{T}^{2}\right)\right) \cap L^{2}\left([0, \infty) ; H^{2}\left(\mathbb{T}^{2}\right)\right)$. By Lemma 2.1, we have $\psi \in L^{\infty}\left([0, \infty) ; L^{2}\left(\mathbb{T}^{1}\right)\right) \cap L^{2}\left([0, \infty) ; H^{2}\left(\mathbb{T}^{1}\right)\right)$. By the triangle and Poincaré's inequalities, it then follows $\Delta \phi \in L^{2}\left([0, \infty) ; L^{2}\left(\mathbb{T}^{2}\right)\right)$. If we further denote

$$
\bar{\phi}=\frac{1}{L_{1} L_{2}} \int_{\mathbb{T}^{2}} \phi(x, y) \mathrm{d} x \mathrm{~d} y=\frac{1}{L_{2}} \int_{\mathbb{T}^{1}}\langle\phi\rangle \mathrm{d} y,
$$

then from (9) we have

$$
\begin{aligned}
\partial_{t} \bar{\phi} & =-\frac{v}{2 L_{1} L_{2}} \int_{\mathbb{T}^{2}}\left|\nabla \phi_{\neq}+\nabla\langle\phi\rangle\right|^{2} \mathrm{~d} x \mathrm{~d} y \\
& =-\frac{v}{2 L_{1} L_{2}} \int_{\mathbb{T}^{2}}\left|\nabla \phi_{\neq}\right|^{2} \mathrm{~d} x \mathrm{~d} y-\frac{v}{2 L_{2}} \int_{\mathbb{T}^{1}}|\psi|^{2} \mathrm{~d} y .
\end{aligned}
$$


By integrating the above equation and applying estimate (22) and (B2), we obtain $\bar{\phi} \in$ $L^{\infty}([0, \infty))$. By Lemma 2.1, it follows that $\psi \in L^{\infty}\left([0, \infty) ; L^{2}\left(\mathbb{T}^{1}\right)\right)$. Hence, $(28)$ and the Poincaré inequality imply that $\langle\phi\rangle \in L^{\infty}\left([0, \infty) ; L^{2}\left(\mathbb{T}^{1}\right)\right)$. As a consequence, $\phi \in L^{\infty}\left([0, \infty) ; L^{2}\left(\mathbb{T}^{2}\right)\right)$. Finally, we note that $\nabla^{2} \phi=\nabla^{2} \phi_{\neq}+\nabla \psi$ so that $\phi \in$ $L^{2}\left([0, \infty) ; H^{2}\left(\mathbb{T}^{2}\right)\right)$. This concludes the proof.

\subsection{Bootstrap estimates}

It remains to establish the bootstrap estimates (B1)-(B2), which we accomplish through a series of lemmas. We address (B2) first.

Lemma 2.2. Let $0<L_{2}<2 \pi$. Assume the bootstrap assumptions (H1) and (H2). There exists $v_{0}=v_{0}\left(\left\|\phi_{\neq}(0)\right\|_{L^{2}}\right)$, explicitly computable, with the following property: for any $0 \leq s \leq t \leq t_{0}$ and for any $v \leq v_{0}$, it holds that

$$
v \int_{s}^{t}\left\|\Delta \phi_{\neq}(\tau)\right\|_{L^{2}}^{2} \mathrm{~d} \tau \leq 2\left\|\phi_{\neq}(s)\right\|_{L^{2}}^{2} .
$$

In particular, (B2) holds.

Proof. The bootstrap assumptions, Lemma 2.1 and the energy estimate (20) give

$$
\begin{aligned}
v \int_{s}^{t}\left\|\Delta \phi_{\neq}(\tau)\right\|_{L^{2}}^{2} \mathrm{~d} \tau & \leq\left\|\phi_{\neq}(s)\right\|_{L^{2}}^{2}+C v \int_{s}^{t}\left\|\phi_{\neq}(\tau)\right\|_{L^{2}}^{6}+\left(1+C_{1}\right)\left\|\phi_{\neq}(\tau)\right\|_{L^{2}}^{2} \mathrm{~d} \tau \\
& \leq\left\|\phi_{\neq}(s)\right\|_{L^{2}}^{2}+\frac{v}{\lambda_{v}} C\left(\left\|\phi_{\neq}(0)\right\|_{L^{2}}^{4}+1+C_{1}\right)\left\|\phi_{\neq}(s)\right\|_{L^{2}}^{2}
\end{aligned}
$$

We observe that, since $v / \lambda_{v} \rightarrow 0$ as $v \rightarrow 0$, there exists $v_{0}$ such that

$$
\frac{\nu_{0}}{\lambda_{\nu_{0}}} \leq \frac{1}{C\left(\left\|\phi_{\neq}(0)\right\|_{L^{2}}^{4}+1+C_{1}\right)} .
$$

Hence, by combining the choice $v_{0}$ with (31) we conclude the proof of the Lemma.

It remains to prove (B1), which we accomplish in different steps. The next lemma states that within a fixed length of time, the quantity $\left\|\phi_{\neq}\right\|_{L^{2}}$ will never grow too fast.

Lemma 2.3. Let $0<L_{2}<2 \pi$. Assume the bootstrap assumptions (H1) and (H2), and fix $\tau^{*}=4 / \lambda_{\nu}$. For any $0 \leq t_{1}<t_{0}$, there exists $v_{0}=v_{0}\left(\left\|\phi_{\neq}(0)\right\|_{L^{2}}\right)$ such that for any $v \leq v_{0}$ there holds

$$
\left\|\phi_{\neq}(t)\right\|_{L^{2}} \leq \sqrt{2}\left\|\phi_{\neq}\left(t_{1}\right)\right\|_{L^{2}} .
$$

for all $t \in\left[t_{1}, t_{1}+\tau^{*}\right] \cap\left[0, t_{0}\right]$. 
Proof. We will assume that $v_{0}$ is small enough so that Lemma 2.2 applies. Again by Lemma 2.1, the energy estimate (20), and the bootstrap assumption (H1), for some positive $C_{2}=C_{2}\left(\left\|\phi_{\neq}(0)\right\|_{L^{2}},\|\psi(0)\|_{L_{y}^{2}}\right)$ we have that

$$
\begin{aligned}
\frac{\mathrm{d}}{\mathrm{d} t}\left\|\phi_{\neq}\right\|_{L^{2}}^{2} & \leq C v\left\|\phi_{\neq}\right\|_{L^{2}}^{6}+C\left(1+C_{1}\right) v\left\|\phi_{\neq}\right\|_{L^{2}}^{2} \\
& \leq C\left(1+C_{1}\right) v\left\|\phi_{\neq}(0)\right\|_{L^{2}}^{2}\left(\left\|\phi_{\neq}\right\|_{L^{2}}^{4}+1\right) \\
& \leq C_{2} v\left(\left\|\phi_{\neq}\right\|_{L^{2}}^{4}+1\right) .
\end{aligned}
$$

Now, we define $T(B)$ as

$$
T(B)=\frac{1}{v C_{2}} \int_{B^{2}}^{2 B^{2}} \frac{\mathrm{d} y}{y^{2}+1}=\frac{1}{v C_{2}}\left(\arctan \left(2 B^{2}\right)-\arctan \left(B^{2}\right)\right) .
$$

It is easy to see that $T(\cdot)$ is a decreasing function and, since by the bootstrap assumption (H1) we have that $\left\|\phi_{\neq}\left(t_{1}\right)\right\|_{L^{2}} \leq 8\left\|\phi_{\neq}(0)\right\|_{L^{2}}$, it follows that $T\left(\left\|\phi_{\neq}\left(t_{1}\right)\right\|_{L^{2}}\right) \geq$ $T\left(8\left\|\phi_{\neq}(0)\right\|_{L^{2}}\right)$. In light of (33), by the definition of $T(B)$ we have that for any $t \in$ $\left[t_{1}, t_{1}+T\left(8\left\|\phi_{\neq}(0)\right\|_{L^{2}}\right)\right] \cap\left[0, t_{0}\right]$, the estimate $\left\|\phi_{\neq}(t)\right\|_{L^{2}} \leq \sqrt{2}\left\|\phi_{\neq}\left(t_{1}\right)\right\|_{L^{2}}$ holds. The lemma is now proved if we choose $\tau^{*} \leq T\left(8\left\|\phi_{\neq}(0)\right\|_{L^{2}}\right)$, which is equivalent to asking for

$$
\frac{v}{\lambda_{\nu}} \leq \frac{1}{4 C_{2}}\left(\arctan \left(128\left\|\phi_{\neq}(0)\right\|_{L^{2}}^{2}\right)-\arctan \left(64\left\|\phi_{\neq}(0)\right\|_{L^{2}}^{2}\right)\right) .
$$

Finally, $v / \lambda_{v} \rightarrow 0$ as $v \rightarrow 0$, so it is enough to satisfy (35) for $v_{0}$. This concludes the proof.

The next lemma shows that by, selecting $v$ sufficiently small, a constant fraction of $\left\|\phi_{\neq}\right\|_{L^{2}}$ decays after a fixed length of time.

Lemma 2.4. Let $0<L_{2}<2 \pi$. Assume the bootstrap assumptions (H1) and (H2), and fix again $\tau^{*}=4 / \lambda_{v}$. If $t_{0} \geq \tau^{*}$ then there exists $v_{0}=v_{0}\left(\left\|\phi_{\neq}(0)\right\|_{L^{2}}\right)$, explicitly computable, with the following property: for any $s \in\left[0, t_{0}-\tau^{*}\right]$ and for any $v \leq v_{0}$,

$$
\left\|\phi_{\neq}\left(\tau^{*}+s\right)\right\|_{L^{2}} \leq \frac{1}{\mathrm{e}}\left\|\phi_{\neq}(s)\right\|_{L^{2}}
$$

Proof. In the course of this proof, we assume that $v_{0}$ is small enough so that Lemma 2.2 can be applied. By the definition of $\tau^{*}$, we have

$$
\left\|\mathcal{S}_{\tau^{*}}\left(\phi_{\neq}(s)\right)\right\|_{L^{2}} \leq \frac{5}{\mathrm{e}^{4}}\left\|\phi_{\neq}(s)\right\|_{L^{2}} \leq \frac{1}{\mathrm{e}^{2}}\left\|\phi_{\neq}(s)\right\|_{L^{2}}
$$


Using this inequality in (13) yields

$$
\begin{aligned}
& \left\|\phi_{\neq}\left(\tau^{*}+s\right)\right\|_{L^{2}} \leq \frac{1}{\mathrm{e}^{2}}\left\|\phi_{\neq}(s)\right\|_{L^{2}} \\
& \quad+C v \int_{s}^{\tau^{*}+s}\left(\left\|\phi_{\neq}\right\|_{L^{2}}^{1 / 2}\left\|\Delta \phi_{\neq}\right\|_{L^{2}}^{3 / 2}+\left\|\Delta \phi_{\neq}\right\|_{L^{2}}+\left\|\phi_{\neq}\right\|_{L^{2}}^{1 / 4}\left\|\Delta \phi_{\neq}\right\|_{L^{2}}^{3 / 4}\left\|\partial_{y}^{2} \psi\right\|_{L_{y}^{2}}\right) \mathrm{d} \tau \\
& \leq \frac{1}{\mathrm{e}^{2}}\left\|\phi_{\neq}(s)\right\|_{L^{2}}+C\left(v \int_{s}^{\tau^{*}+s}\left\|\phi_{\neq}(\tau)\right\|_{L^{2}}^{2} \mathrm{~d} \tau\right)^{1 / 4}\left(v \int_{s}^{\tau^{*}+s}\left\|\Delta \phi_{\neq}(\tau)\right\|_{L^{2}}^{2} \mathrm{~d} \tau\right)^{3 / 4} \\
& \quad+C\left(v \int_{s}^{\tau^{*}+s}\left\|\Delta \phi_{\neq}(\tau)\right\|_{L^{2}}^{2} \mathrm{~d} \tau\right)^{1 / 2}\left(v \tau^{*}\right)^{1 / 2} \\
& \quad+C\left(v \int_{s}^{\tau^{*}+s}\left\|\Delta \phi_{\neq}(\tau)\right\|_{L^{2}}^{2} \mathrm{~d} \tau\right)^{3 / 8}\left(v \int_{s}^{\tau^{*}+s}\left\|\partial_{y}^{2} \psi(\tau)\right\|_{L^{2}}^{2} \mathrm{~d} \tau\right)^{1 / 2} \\
& \quad\left(v \int_{s}^{\tau^{*}+s}\left\|\phi_{\neq}(\tau)\right\|_{L^{2}}^{2} \mathrm{~d} \tau\right)^{1 / 8} \cdot
\end{aligned}
$$

Using the bootstrap assumptions (H1)-(H2) and Lemma 2.1, it then follows that

$$
\begin{aligned}
\left\|\phi_{\neq}\left(\tau^{*}+s\right)\right\|_{L^{2} \leq} \leq & \frac{1}{\mathrm{e}^{2}}\left\|\phi_{\neq}(s)\right\|_{L^{2}}+C\left(v \tau^{*}\right)^{1 / 4}\left\|\phi_{\neq}(s)\right\|_{L^{2}}^{2}+C\left(v \tau^{*}\right)^{1 / 2}\left\|\phi_{\neq}(s)\right\|_{L^{2}} \\
& +C \sqrt{C_{1}}\left(v \tau^{*}\right)^{1 / 8}\left\|\phi_{\neq}(s)\right\|_{L^{2}} \\
\leq & \frac{1}{\mathrm{e}^{2}}\left\|\phi_{\neq}(s)\right\|_{L^{2}}+C\left(v \tau^{*}\right)^{1 / 8}\left(\left\|\phi_{\neq}(s)\right\|_{L^{2}}+\sqrt{C_{1}}\right)\left\|\phi_{\neq}(s)\right\|_{L^{2}},
\end{aligned}
$$

where we used the fact that $\nu \tau^{*} \ll 1$ when $\nu_{0}$ is small enough. By further restricting $v_{0}$ so that

$$
\frac{1}{\mathrm{e}^{2}}+C\left(4 v_{0} \lambda_{\nu_{0}}^{-1}\right)^{1 / 8}\left(8\left\|\phi_{\neq}(0)\right\|_{L^{2}}+\sqrt{C_{1}}\right) \leq \frac{1}{\mathrm{e}}
$$

the desired result follows from (39).

Now we are ready to show that the bootstrap assumption (H1) can be refined.

Lemma 2.5. Let $0<L_{2}<2 \pi$. Assume the bootstrap assumptions (H1) and (H2). There exists $v_{0}=v_{0}\left(\left\|\phi_{\neq}(0)\right\|_{L^{2}}\right)$, explicitly computable, with the following property: for any $0 \leq s \leq t \leq t_{0}$ and for any $v \leq v_{0}$, it holds that

$$
\left\|\phi_{\neq}(t)\right\|_{L^{2}} \leq 4 \mathrm{e}^{-\lambda_{v}(t-s) / 4}\left\|\phi_{\neq}(s)\right\|_{L^{2}} .
$$

In particular, (B1) holds.

Proof. We fix $v_{0}=v_{0}\left(\left\|\phi_{\neq}(0)\right\|_{L^{2}}\right)$ so that all the restrictions in Lemmata 2.2-2.4 are fulfilled. If $t_{0}<\tau^{*}$ then (B1) directly follows by Lemma 2.3 since $\sqrt{2} \mathrm{e}<4$. When $t_{0} \geq \tau^{*}$, by Lemma 2.4 , we have

$$
\left\|\phi_{\neq}\left(n \tau^{*}+s\right)\right\|_{L^{2}} \leq \mathrm{e}^{-n}\left\|\phi_{\neq}(s)\right\|_{L^{2}}, \quad \text { for any } n \in \mathbb{Z}_{+} \text {satisfying } s+n \tau^{*} \leq t_{0} .
$$


For any $0 \leq s \leq t \leq t_{0}$, there exists $n$ such that $t \in\left[n \tau^{*}+s,(n+1) \tau^{*}+s\right)$. From Lemma 2.3 with $t_{1}=n \tau^{*}+s$, it follows that

$$
\begin{aligned}
& \left\|\phi_{\neq}(t)\right\|_{L^{2}} \leq \sqrt{2}\left\|\phi_{\neq}\left(n \tau^{*}+s\right)\right\|_{L^{2}} \leq \sqrt{2} \mathrm{e}^{-n}\left\|\phi_{\neq}(s)\right\|_{L^{2}} \\
& \leq \sqrt{2} \mathrm{e}^{1-(t-s) / \tau^{*}}\left\|\phi_{\neq}(s)\right\|_{L^{2}} \leq 4 \mathrm{e}^{-\lambda_{v}(t-s) / 4}\left\|\phi_{\neq}(s)\right\|_{L^{2}} .
\end{aligned}
$$

This concludes the proof of the lemma.

\section{Semigroup estimates}

In this section, we prove Proposition 1.2, namely an improved decay estimate for the semigroup generated by $H_{v}$ in $L^{2}$, under a general condition on the shear velocity profile $u$.

We denote by $\stackrel{\circ}{L}^{2}\left(\mathbb{T}^{2}\right)$ the closed subspace of $L^{2}\left(\mathbb{T}^{2}\right)$ of elements for which $\langle g\rangle=0$. By Fubini-Tonelli's Theorem, such elements are also mean-zero on the torus. We will be concerned with the restriction of the operator $H_{v}$ to $\stackrel{\circ}{L}^{2}\left(\mathbb{T}^{2}\right)$ viewed as an unbounded operator. By slight abuse of notation, we denote the restriction also by $H_{\nu}$. It is straightforward to check that the projection onto $\stackrel{\circ}{L}^{2}\left(\mathbb{T}^{2}\right)$ commutes with the semigroup $\mathrm{e}^{-t H_{v}}$ generated by $H_{v}$.

Let $(X,\|\cdot\|)$ be a complex Hilbert space and let $H$ be a closed, densely defined operator on $X$. As shown in [39], if $H$ is an $m$-accretive operator on $X$, then the decay properties of the semigroup $\mathrm{e}^{-t H}$ can be understood in terms of the following quantity:

$$
\Psi(H)=\inf \{\|(H-i \lambda) g\|: g \in D(H), \lambda \in \mathbb{R},\|g\|=1\},
$$

is related to the pseudospectral properties of the operator [18]. Following [39], for $\frac{L_{1} k}{2 \pi} \in \mathbb{Z} \backslash\{0\}$ and $v \in(0,1]$, we consider the operator $H_{v}$ localized to the $k$ th Fourier mode in the direction of the shear, namely, the operator

$$
H_{v, k}=v \Delta_{k}^{2}+i k u(y), \quad \Delta_{k}:=-k^{2}+\partial_{y y} .
$$

Following the arguments in [39] for the Laplace operator, it can be shown that $H_{\nu, k}$ is an $m$-accretive operator on $L^{2}\left(\mathbb{T}^{1}\right)$ with domain $H^{4}\left(\mathbb{T}^{1}\right)$. Here, $L^{2}$ is a space of complex-valued functions. Then, as a consequence of [39]*Theorem 1.3,

$$
\left\|\mathrm{e}^{-H_{\nu, k} t}\right\|_{\text {op }} \leq \mathrm{e}^{-t \Psi\left(H_{v, k}\right)+\pi / 2}, \quad \forall t \geq 0,
$$

where $\|\cdot\|_{\text {op }}$ denotes the operator norm. To establish lower bounds on $\Psi\left(H_{\nu, k}\right)$, we assume the following condition on the shear flow.

Assumption 3.1. There exist $m, N \in \mathbb{N}, c_{1}>0$ and $\delta_{0} \in\left(0, L_{2}\right)$ with the property that, for any $\lambda \in \mathbb{R}$ and any $\delta \in\left(0, \delta_{0}\right)$, there exist $n \leq N$ and points $y_{1}, \ldots y_{n} \in$ $\left[0, L_{2}\right)$ such that

$$
|u(y)-\lambda| \geq c_{1}\left(\frac{\delta}{L_{2}}\right)^{m}, \quad \forall\left|y-y_{j}\right| \geq \delta, \quad \forall j \in\{1, \ldots n\} .
$$


Remark 3.1. Assumption 3.1 is heavily inspired by a similar property of the velocity field associated to the Oseen's vortex [33]. In [20], Gallay previously observed that the method of proof in [33] can be extended to more general shear flows assuming a condition similar to (47). We observe that a strictly monotone shear flow satisfies Assumption 3.1 with $m=1$. However, no such flows exists on the torus $\mathbb{T}^{2}$, where any shear flow must have at least a simple critical point, so that we always have $m \geq 2$. See for instance the example in Proposition 1.2.

The following is the main result of this section.

Proposition 1.1. Let $u$ satisfy Assumption 3.1. Assume $k \neq 0$ and $v|k|^{-1} \leq 1$. There exists a constant $\varepsilon_{0}^{\prime}>0$, independent of $v$ and $k$, such that

$$
\Psi\left(H_{v, k}\right) \geq \varepsilon_{0}^{\prime} v^{\frac{m}{m+4}}|k|^{\frac{4}{m+4}} .
$$

We state next a direct consequence of the theorem.

Corollary 3.1. In the hypotheses of Proposition 1.1 , let $P_{k}$ denote the $L^{2}$ projection onto the $k$-th Fourier mode in the horizontal direction. Then, for every $t \geq 0$,

$$
\left\|\mathrm{e}^{-H_{\nu} t} P_{k}\right\|_{o p} \leq \mathrm{e}^{-\varepsilon_{0}^{\prime} v^{\frac{m}{m+4}}|k|^{\frac{4}{m+4}} t+\pi / 2} .
$$

In particular, $H_{v}$ generates an exponentially stable semigroup in $\stackrel{\circ}{L}^{2}\left(\mathbb{T}^{2}\right)$ with rate:

$$
\left\|\mathrm{e}^{-H_{\nu} t}\right\|_{o p} \leq \mathrm{e}^{-\lambda_{\nu}^{\prime} t+\pi / 2}, \quad t>0
$$

where $\lambda_{v}^{\prime}=\varepsilon_{0}^{\prime} \nu^{\frac{m}{m+4}}$ for some $\varepsilon_{0}^{\prime}>0$.

Before proving Proposition 1.1, we show that the Assumption 3.1 is satisfied with $m \geq 2$ for $u$ as in (7).

Example 3.1. We consider the case of $u(y)=(\sin (y))^{\ell}, \ell \in \mathbb{N}$, defined on $[0,2 \pi)$. For a general period $L_{2}$, the result follows by a standard rescaling argument. Without loss of generality, we may assume that $\delta_{0}>0$ is small enough so that $\cos \left(\delta_{0}\right) \geq 1 / 2$. In particular, $\delta_{0}$ and hence $\delta<1$ and, for every $C \geq 1$,

$$
\sin (\delta / C) \geq \frac{\delta}{2 C}, \quad \forall \delta \in\left(0, \delta_{0}\right) .
$$

Given $\lambda \in \mathbb{R}$, we choose the set of points $y_{1}, \ldots, y_{n}$ to be the union of the set of the critical points of $u$ with the set $u^{-1}(\{\lambda\})$ (possibly empty). More precisely, we consider

$$
Y:=\left\{\begin{array}{ll}
\{\pi / 2,3 \pi / 2\} \cup u^{-1}(\{\lambda\}), & \ell=1, \\
\{0, \pi / 2, \pi, 3 \pi / 2\} \cup u^{-1}(\{\lambda\}), & \ell \geq 2,
\end{array} \quad n:=|Y| .\right.
$$

There are at most $N=8$ points in $Y$. Observe that the critical points at $y=\pi / 2,3 \pi / 2$ are such that $u^{\prime \prime}(\pi / 2), u^{\prime \prime}(3 \pi / 2) \neq 0$ for any $\ell \geq 1$. On the other hand, for $\ell \geq 2$ 
the critical points at $y=0, \pi$ are such that $u^{(j)}(0)=u^{(j)}(\pi)=0$ for every $j=$ $1, \ldots, \ell-1$ and $u^{(\ell)}(0), u^{(\ell)}(\pi) \neq 0$. We denote $Y=\left\{y_{i}\right\}_{i=1}^{n}$ and we order the points in such a way that $0 \leq y_{i} \leq y_{i+1} \leq 2 \pi$ for $i=1, \ldots, N$. We fix $\delta \in\left(0, \delta_{0}\right)$ and consider different cases.

Case $\lambda \in[0,1]$. Denote by $y_{\lambda}$ the smallest element of $u^{-1}(\{\lambda\})$. Due to the symmetries of $u$, we know that $y_{\lambda} \in[0, \pi / 2]$. We consider three situations.

- $0 \leq y_{\lambda}<\delta / 4$ : In this case, thanks to (51), we have

$$
\begin{aligned}
|u(y)-\lambda| & =\left|(\sin (y))^{\ell}-\left(\sin \left(y_{\lambda}\right)\right)^{\ell}\right| \geq|\sin (\delta)|^{\ell}-\left|\sin \left(y_{\lambda}\right)\right|^{\ell} \\
& \geq \frac{\delta^{\ell}}{2^{\ell}}-|\sin (\delta / 4)|^{\ell} \geq \frac{\delta^{\ell}}{2^{\ell}}-\frac{\delta^{\ell}}{4^{\ell}} \geq \frac{\delta^{\ell}}{2^{\ell+1}},
\end{aligned}
$$

where we used that $0 \leq \sin (y) \leq y$.

- $\left|y_{\lambda}-\pi / 2\right|<\delta / 4$ : In this case we have

$$
\begin{aligned}
|u(y)-\lambda| & =\left|(\sin (y))^{\ell}-\left(\sin \left(y_{\lambda}\right)\right)^{\ell}\right| \geq\left|(\sin (\pi / 2-\delta))^{\ell}-\left(\sin \left(y_{\lambda}\right)\right)^{m}\right| \\
& \left.\left.\left.\geq \mid(\sin (\pi / 2-\delta)))^{\ell}-(\sin (\pi / 2))\right)^{\ell}|-|(\sin (\pi / 2))\right)^{\ell}-(\sin (\pi / 2-\delta / 4))\right)^{\ell} \mid .
\end{aligned}
$$

Since $\partial_{y}(\sin (y))^{\ell}=\ell(\sin (y))^{\ell-1} \cos (y)$ and $\partial_{y}^{2}(\sin (y))^{\ell}=\ell(\ell-1)(\sin (y))^{\ell-2}$ $\cos ^{2}(y)-\ell(\sin (y))^{\ell}$,

$\left.\mid(\sin (\pi / 2-\delta)))^{\ell}-(\sin (\pi / 2))\right)^{\ell}|=|\left(1-\frac{\delta^{2}}{2}+O\left(\delta^{4}\right)\right)^{e} l l-1 \mid=\frac{\ell}{2} \delta^{2}+O\left(\delta^{4}\right)$.

so that, by possibly restricting the size of $\delta_{0}$ further (depending on $\ell$ ), we also have

$$
\left.\mid(\sin (\pi / 2-\delta)))^{\ell}-(\sin (\pi / 2))\right)^{\ell} \mid \geq \frac{\ell}{4} \delta^{2}
$$

On the other hand,

$$
\left.\mid(\sin (\pi / 2)))^{\ell}-(\sin (\pi / 2-\delta / 4))\right)^{\ell} \mid \leq \frac{\ell}{8} \delta^{2} .
$$

Consequently,

$$
|u(y)-\lambda| \geq \frac{\ell}{8} \delta^{2} .
$$

- $y_{\lambda} \geq \delta / 4$ and $\left|y_{\lambda}-\pi / 2\right| \geq \delta / 4$ : In this case, we have

$$
\begin{aligned}
|u(y)-\lambda| & =\left|(\sin (y))^{\ell}-\left(\sin \left(y_{\lambda}\right)\right)^{\ell}\right| \\
& \geq \min \left\{\left|\left(\sin \left(y_{\lambda}+\delta / 8\right)\right)^{\ell}-\left(\sin \left(y_{\lambda}\right)\right)^{\ell}\right|,\left|\left(\sin \left(y_{\lambda}-\delta / 8\right)\right)^{\ell}-\left(\sin \left(y_{\lambda}\right)\right)^{\ell}\right|\right\} .
\end{aligned}
$$


We observe that $y_{\lambda}+\delta / 8<\pi / 2-\delta / 8$ and $y_{\lambda}-\delta / 8>\delta / 8$. Then if $y_{\lambda} \geq \pi / 4$, it follows by the Mean Value Theorem that for some $\xi \in\left(y_{\lambda}, y_{\lambda}+\delta / 8\right)$,

$$
\begin{aligned}
\left|\left(\sin \left(y_{\lambda}+\delta / 8\right)\right)^{\ell}-\left(\sin \left(y_{\lambda}\right)\right)^{\ell}\right| & =\ell\left|(\sin (\xi))^{\ell-1} \cos (\xi)\right| \frac{\delta}{8} \\
& \geq \ell\left|(\sin (\pi / 4))^{\ell-1} \cos (\pi / 2-\delta / 8)\right| \frac{\delta}{8} \\
& \geq \frac{\ell}{2^{\frac{\ell-1}{2}}}|\sin (\delta / 8)| \frac{\delta}{8} \geq \frac{\ell}{2^{\frac{\ell-1}{2}}} \frac{\delta^{2}}{128},
\end{aligned}
$$

also using (51). Otherwise, if $y_{\lambda} \leq \pi / 4$, we have

$$
\begin{aligned}
\left|\left(\sin \left(y_{\lambda}+\delta / 8\right)\right)^{\ell}-\left(\sin \left(y_{\lambda}\right)\right)^{\ell}\right| & =\ell\left|(\sin (\xi))^{\ell-1} \cos (\xi)\right| \frac{\delta}{8} \\
& \geq \ell\left|(\sin (\delta / 4))^{\ell-1} \cos (\pi / 4+\delta / 8)\right| \frac{\delta}{8} \\
& \geq \ell\left|(\sin (\delta / 4))^{\ell-1} \cos (\pi / 3)\right| \frac{\delta}{8} \geq \frac{\ell}{8^{\ell+1}} \delta^{\ell} .
\end{aligned}
$$

A lower bound for $\left.\left.\mid\left(\sin \left(y_{\lambda}-\delta / 8\right)\right)\right)^{\ell}-\left(\sin \left(y_{\lambda}\right)\right)\right)^{\ell} \mid$ can be proved in a similar way.

Case $\lambda>1$. In this case $u^{-1}(\{\lambda\})=\emptyset$. Hence, since $\operatorname{dist}(y, Y) \geq \delta$, we simply have

$$
\begin{aligned}
|u(y)-\lambda| & \geq\left|(\sin (y))^{\ell}-(\sin (\pi / 2))^{\ell}\right| \geq\left|(\sin (\pi / 2-\delta))^{\ell}-(\sin (\pi / 2))^{\ell}\right| \\
& \geq \ell\left|(\sin (\xi))^{\ell-1} \cos (\xi)\right| \delta \geq \ell\left|(\sin (\pi / 6))^{\ell-1} \cos (\pi / 2-\delta)\right| \delta \geq \frac{\ell}{2^{\ell}} \delta^{2},
\end{aligned}
$$

where we used (51) in the last inequality.

Case $\lambda<0$. This case is only relevant for $\ell$ even, hence $\ell \geq 2$. In fact, for $\ell$ odd we proceed as for the case $\lambda \in[0,1]$ by symmetry. Here, again $u^{-1}(\{\lambda\})=\emptyset$. Hence, since $\operatorname{dist}(y, Y) \geq \delta$, we have

$$
|u(y)-\lambda| \geq\left|(\sin (y))^{\ell}\right| \geq(\sin (\delta))^{\ell} \geq \frac{\delta^{\ell}}{2^{\ell}}
$$

by $(51)$.

Since $\delta>\delta^{2}$, combining all these estimates together gives that there exists a constant $c_{\ell}>0$ such that

$$
|u(y)-\lambda| \geq c_{\ell} \delta^{\max \{\ell, 2\}},
$$

as we wanted.

We now turn our attention to the proof of Proposition 1.1.

Proof of Proposition 1.1. The theorem follows by establishing a lower bound on $\Psi\left(H_{\nu, k}\right)$. In the following, $\|\cdot\|$ denotes the $L^{2}$ norm and $\langle\cdot, \cdot\rangle$ denotes the Hermitian inner product in $L^{2}$. 
We fix $\lambda \in \mathbb{R}$ and pick $g \in D\left(H_{\nu, k}\right)$ with $\|g\|=1$. For notational convenience, we set

$$
H:=H_{v, k}-i k \lambda=v \Delta_{k}^{2}+i k(u(y)-\lambda) .
$$

Let $\chi:\left[0, L_{2}\right) \rightarrow[-1,1]$ be a smooth approximation of $\operatorname{sign}(u(y)-\lambda)$ such that $\left\|\chi^{\prime}\right\|_{L^{\infty}} \leq c_{2} \delta^{-1},\left\|\chi^{\prime \prime}\right\|_{L^{\infty}} \leq c_{2} \delta^{-2}, \chi(u-\lambda) \geq 0$ and

$$
\chi(y)(u(y)-\lambda)=|u(y)-\lambda|, \quad \text { whenever }\left|y-y_{j}\right| \geq \delta, \quad \forall j \in\{1, \ldots n\},
$$

where $y_{j}$ are the points in Assumption 3.1. The function $\chi$ can be constructed via a standard mollification argument. A double integration by parts in $y$ yields the identity

$$
\operatorname{Re}\langle H g, g\rangle=v\left\|\Delta_{k} g\right\|^{2}
$$

which implies that

$$
\left\|\Delta_{k} g\right\|^{2} \leq \frac{1}{v}\|H g\|\|g\|
$$

On the other hand, we have

$$
\begin{aligned}
\langle H g, \chi g\rangle= & v\left\langle\Delta_{k}^{2} g, \chi g\right\rangle+i k\langle(u(y)-\lambda) g, \chi g\rangle=v\left\langle\Delta_{k} g, \chi^{\prime \prime} g\right\rangle \\
& +2 v\left\langle\Delta_{k} g, \chi^{\prime} \partial_{y} g\right\rangle+v\left\langle\Delta_{k} g, \chi \Delta_{k} g\right\rangle+i k\langle(u(y)-\lambda) g, \chi g\rangle
\end{aligned}
$$

so that

$$
\operatorname{Im}\langle H g, \chi g\rangle=v \operatorname{Im}\left\langle\Delta_{k} g, \chi^{\prime \prime} g\right\rangle+2 v \operatorname{Im}\left\langle\Delta_{k} g, \chi^{\prime} \partial_{y} g\right\rangle+k\langle(u(y)-\lambda) g, \chi g\rangle .
$$

In particular, from the properties of the function $\chi$ and the interpolation inequality $\left\|\partial_{y} g\right\|^{2} \leq\left\|\Delta_{k} g\right\|\|g\|$, it follows that

$$
|k|\langle(u(y)-\lambda) g, \chi g\rangle \leq\|H g\|\|g\|+\frac{c_{2} v}{\delta^{2}}\left\|\Delta_{k} g\right\|\|g\|+\frac{c_{2} v}{\delta}\left\|\Delta_{k} g\right\|^{3 / 2}\|g\|^{1 / 2} .
$$

We denote

$$
E:=\left\{y \in\left[0, L_{2}\right):\left|y-y_{j}\right| \geq \delta, \text { for } j=1, \ldots, n\right\}
$$

where $y_{j}$ are the points in Assumption 3.1. By (47) we have

$$
\langle(u(y)-\lambda) g, \chi g\rangle \geq \int_{E}|u(y)-\lambda||g(y)|^{2} \mathrm{~d} y \geq c_{1}\left(\frac{\delta}{L_{2}}\right)^{m} \int_{E}|g(y)|^{2} \mathrm{~d} y .
$$


Utilizing (67) and (70), we find that there exists a positive constant $\tilde{c}_{2}$ such that

$$
\begin{aligned}
& \int_{E}|g(y)|^{2} \mathrm{~d} y \leq \frac{1}{c_{1}|k|}\left(\frac{L_{2}}{\delta}\right)^{m}\left[\|H g\|\|g\|+\frac{c_{2} v}{\delta^{2}}\left\|\Delta_{k} g\right\|\|g\|+\frac{c_{2} v}{\delta}\left\|\Delta_{k} g\right\|^{3 / 2}\|g\|^{1 / 2}\right] \\
& \leq \frac{1}{c_{1}|k|}\left(\frac{L_{2}}{\delta}\right)^{m}\|H g\|\|g\|+\tilde{c}_{2}\left(\left(\frac{v}{|k| \delta^{2}}\right)^{2}\left(\frac{L_{2}}{\delta}\right)^{2 m}\right. \\
& \left.\quad+\left(\frac{v}{|k| \delta}\right)^{4 / 3}\left(\frac{L_{2}}{\delta}\right)^{\frac{4 m}{3}}\right)\left\|\Delta_{k} g\right\|^{2}+\frac{1}{4}\|g\|^{2} \\
& \leq\left(\frac{1}{c_{1}|k|}\left(\frac{L_{2}}{\delta}\right)^{m}+\frac{\tilde{c}_{2}}{v}\left(\left(\frac{v}{|k| L_{2}^{2}}\right)^{2}\left(\frac{L_{2}}{\delta}\right)^{2 m+4}\right.\right. \\
& \left.\left.\quad+\left(\frac{v}{|k| L_{2}}\right)^{4 / 3}\left(\frac{L_{2}}{\delta}\right)^{\frac{4}{3}(m+1)}\right)\right)\|H g\|\|g\|+\frac{1}{4}\|g\|^{2} .
\end{aligned}
$$

On the other hand, since $E^{c}$ is of size at most $N \delta$, we have

$$
\begin{aligned}
\int_{E^{c}}|g(y)|^{2} \mathrm{~d} y \leq N \delta\|g\|_{L^{\infty}}^{2} & \leq N \delta\left(2\|g\|\left\|\partial_{y} g\right\|+\frac{1}{L_{2}}\|g\|^{2}\right) \\
& \leq N \delta\left(2\|g\|^{3 / 2}\left\|\Delta_{k} g\right\|^{1 / 2}+\frac{1}{L_{2}}\|g\|^{2}\right) \\
& \leq \frac{(6 N \delta)^{4}}{12}\left\|\Delta_{k} g\right\|^{2}+\left(\frac{N \delta}{L_{2}}+\frac{1}{4}\right)\|g\|^{2} \\
& \leq \frac{(6 N \delta)^{4}}{12 v}\|H g\|\|g\|+\left(\frac{N \delta}{L_{2}}+\frac{1}{4}\right)\|g\|^{2}
\end{aligned}
$$

where we made use of (67) in the last inequality. Without loss of generality, we can assume that $\delta_{0}$ in Assumption 3.1 is small enough so that $\delta \leq L_{2} /(4 N)$ for all $\delta \in\left(0, \delta_{0}\right)$. Thus we can add (73) and (74) to conclude that

$$
\begin{aligned}
\|g\| \leq & 4\left(\frac{1}{c_{1}|k|}\left(\frac{L_{2}}{\delta}\right)^{m}+\tilde{c}_{2} \frac{v}{\left(|k| L_{2}^{2}\right)^{2}}\left(\frac{L_{2}}{\delta}\right)^{2 m+4}\right. \\
& \left.+\tilde{c}_{2} \frac{v^{1 / 3}}{\left(|k| L_{2}\right)^{4 / 3}}\left(\frac{L_{2}}{\delta}\right)^{\frac{4}{3}(m+1)}+\frac{(6 N \delta)^{4}}{12 v}\right)\|H g\| .
\end{aligned}
$$

We now take $\delta$ satisfying

$$
\frac{\delta}{L_{2}}=c_{3}\left(\frac{v}{|k|}\right)^{\frac{1}{m+4}}
$$

for some sufficiently small constant $c_{3}>0$, which is independent of $v, k$ since $v|k|^{-1} \leq 1$. We conclude that

$$
v^{\frac{m}{m+4}}|k|^{\frac{4}{m+4}}=v^{\frac{m}{m+4}}|k|^{\frac{4}{m+4}}\|g\| \leq c_{4}\|H g\|,
$$


for a large enough constant $c_{4}>0$ independent of $v$ and $k$. By definition then

$$
\Psi(H) \geq \varepsilon_{0}^{\prime} v^{\frac{m}{m+4}}|k|^{\frac{4}{m+4}},
$$

for some constant $\varepsilon_{0}^{\prime}>0$ independent of $v$ and $k$, whence proving the proposition.

Remark 3.2. The proof of Proposition 1.1 carries over to the slightly more general case of the semigroup generated by the hypoelliptic operator

$$
\widetilde{H}_{v}=u(y) \partial_{x}+v \partial_{y}^{4}
$$

for which the same semigroup estimate (50) holds.

\section{Acknowledgements}

The authors thank Tarek Elgindi and Thierry Gallay for insightful discussions.

Open Access. This article is licensed under a Creative Commons Attribution 4.0 International License, which permits use, sharing, adaptation, distribution and reproduction in any medium or format, as long as you give appropriate credit to the original author(s) and the source, provide a link to the Creative Commons licence, and indicate if changes were made. The images or other third party material in this article are included in the article's Creative Commons licence, unless indicated otherwise in a credit line to the material. If material is not included in the article's Creative Commons licence and your intended use is not permitted by statutory regulation or exceeds the permitted use, you will need to obtain permission directly from the copyright holder. To view a copy of this licence, visit http://creativecommons.org/licenses/ by/4.0/.

Publisher's Note Springer Nature remains neutral with regard to jurisdictional claims in published maps and institutional affiliations.

\section{REFERENCES}

[1] D. M. Ambrose and A. L. Mazzucato, Global existence and analyticity for the 2D KuramotoSivashinsky equation, J. Dynam. Differential Equations, 31 (2019), no. 3, 1525-1547.

[2] D. M. Ambrose and A. L. Mazzucato, Global solutions of the two-dimensional KuramotoSivashinsky equation with a linearly growing mode in each direction, J. Nonlinear Sci. 31 (2021), no. 6, Paper No. 96.

[3] M. Beck and C. E. Wayne, Metastability and rapid convergence to quasi-stationary bar states for the two-dimensional Navier-Stokes equations, Proc. Roy. Soc. Edinburgh Sect. A 143 (2013), no. 5, 905-927.

[4] J. Bedrossian and M. Coti Zelati, Enhanced dissipation, hypoellipticity, and anomalous small noise inviscid limits in shear flows, Arch. Ration. Mech. Anal. 224 (2017), no. 3, 1161-1204.

[5] J. Bedrossian and S. He, Suppression of blow-up in Patlak-Keller-Segel via shear flows, SIAM J. Math. Anal. 49 (2017), no. 6, 4722-4766.

[6] S. Benachour, I. Kukavica, W. Rusin, and M. Ziane, Anisotropic estimates for the two-dimensional Kuramoto-Sivashinsky equation, J. Dynam. Differential Equations 26 (2014), no. 3, 461-476.

[7] A. Biswas and D. Swanson, Existence and generalized Gevrey regularity of solutions to the Kuramoto-Sivashinsky equation in $\mathbb{R}^{n}$, J. Differential Equations 240 (2007), no. 1, 145-163.

[8] J. C. Bronski and T. N. Gambill, Uncertainty estimates and $L_{2}$ bounds for the Kuramoto-Sivashinsky equation, Nonlinearity 19 (2006), no. 9, 2023-2039. 
[9] P. Collet, J.-P. Eckmann, H. Epstein, and J. Stubbe, Analyticity for the Kuramoto-Sivashinsky equation, Phys. D 67 (1993), no. 4, 321-326.

[10] M. Colombo, M. Coti Zelati, and K. Widmayer, Mixing and diffusion for rough shear flows, arXiv e-prints (Sep. 2020), available at 2009.12268.

[11] P. Constantin, A. Kiselev, L. Ryzhik, and A. Zlatošs, Diffusion and mixing in fluid flow, Ann. of Math. (2) 168 (2008), no. 2, 643-674.

[12] M. Coti Zelati, M. G. Delgadino, and T. M. Elgindi, On the relation between enhanced dissipation timescales and mixing rates, Comm. Pure Appl. Math. 73 (2020), no. 6, 1205-1244.

[13] M. Coti Zelati and M. Dolce, Separation of time-scales in drift-diffusion equations on $\mathbb{R}^{2}$, J. Math. Pures Appl. 142 (2020), 58-75.

[14] M. Coti Zelati, T. M. Elgindi, and K. Widmayer, Enhanced dissipation in the Navier-Stokes equations near the Poiseuille flow, Comm. Math. Phys. 378 (2020), no. 2, 987-1010.

[15] A. Dandekar and L. R. Collins, Effect of nonunity Lewis number on premixed flame propagation through isotropic turbulence, Combustion and Flame 101 (1995), no. 4, 428-440.

[16] Y. Feng and G. Iyer, Dissipation enhancement by mixing, Nonlinearity 32 (2019), no. 5, 1810-1851.

[17] Y. Feng and A. L. Mazzucato, Global existence for the two-dimensional Kuramoto-Sivashinsky equation with advection, 2020-09, arXiv e-prints.

[18] I. Gallagher, T. Gallay, and F. Nier, Spectral asymptotics for large skew-symmetric perturbations of the harmonic oscillator, Int. Math. Res. Not. IMRN 12 (2009), 2147-2199.

[19] T. Gallay, Enhanced dissipation and axisymmetrization of two-dimensional viscous vortices, Arch. Ration. Mech. Anal. 230 (2018), no. 3, 939-975.

[20] T. Gallay, Estimations pseudo-spectrales et stabilité des tourbillons plans, Séminaire BOURBAKI (2019), 72e.

[21] L. Giacomelli, F. Otto, New bounds for the Kuramoto-Sivashinsky equation, Comm. Pure Appl. Math. 58 (2005), no. 3, 297-318.

[22] M. Goldman, M. Josien, and F. Otto, New bounds for the inhomogenous Burgers and the KuramotoSivashinsky equations, Comm. Partial Differential Equations 40 (2015), no. 12, 2237-2265.

[23] D. Goluskin and G. Fantuzzi, Bounds on mean energy in the Kuramoto-Sivashinsky equation computed using semidefinite programming, Nonlinearity 32 (2019), no. 5, 1705-1730.

[24] Z. Grujić, Spatial analyticity on the global attractor for the Kuramoto-Sivashinsky equation, J. Dynam. Differential Equations 12 (2000), no. 1, 217-228.

[25] S. He and E. Tadmor, Suppressing chemotactic blow-up through a fast splitting scenario on the plane, Arch. Ration. Mech. Anal. 232 (2019), no. 2, 951-986.

[26] J. M. Hyman and B. Nicolaenko, The Kuramoto-Sivashinsky equation: a bridge between PDEs and dynamical systems, 1986, pp. 113-126. Solitons and coherent structures (Santa Barbara, Calif., 1985).

[27] X. Ioakim and Y.-S. Smyrlis, Analyticity for Kuramoto-Sivashinsky-type equations in two spatial dimensions, Math. Methods Appl. Sci. 39 (2016), no. 8, 2159-2178.

[28] G. Iyer, X. Xu, and A. Zlatoš, Convection-Induced Singularity Suppression in the Keller-Segel and Other Non-linear PDEs, Trans. Amer. Math. Soc. 374 (2021), no. 9, 6039-6058.

[29] A. Kiselev and X. Xu, Suppression of chemotactic explosion by mixing, Arch. Ration. Mech. Anal. 222 (2016), no. 2, 1077-1112.

[30] I. Kukavica and D. Massatt, On the global existence for the Kuramoto-Sivashinsky equation, J. Dynam. Differential Equations (2021).

[31] Y. Kuramoto, Diffusion-Induced Chaos in Reaction Systems, Progress of Theoretical Physics Supplement, 64 (197802), 346-367, https://academic.oup.com/ptps/article-pdf/doi/10.1143/PTPS.64. 346/5293041/64-346.pdf.

[32] A. Larios and K. Yamazaki, On the well-posedness of an anisotropically-reduced two-dimensional Kuramoto-Sivashinsky equation, Phys. D 411 (2020), 132560, 14.

[33] T. Li, D. Wei, and Z. Zhang, Pseudospectral and spectral bounds for the Oseen vortices operator, Ann. Sci. Éc. Norm. Supér., 53 (2020), no. 4, 993-1035.

[34] B. Nicolaenko, B. Scheurer, and R. Temam, Some global dynamical properties of the KuramotoSivashinsky equations: nonlinear stability and attractors, Phys. D 16 (1985), no. 2, 155-183.

[35] F. Otto, Optimal bounds on the Kuramoto-Sivashinsky equation, J. Funct. Anal. 257 (2009), no. 7, 2188-2245. 
[36] G. R. Sell and M. Taboada, Local dissipativity and attractors for the Kuramoto-Sivashinsky equation in thin 2D domains, Nonlinear Anal. 18 (1992), no. 7, 671-687.

[37] G. I. Sivashinsky, Nonlinear analysis of hydrodynamic instability in laminar flames-i. derivation of basic equations, Acta Astronautica 4 (1977), no. 11, 1177-1206.

[38] M. E. Taylor, Partial differential equations III. Nonlinear equations, Second, Applied Mathematical Sciences, vol. 117, Springer, New York, 2011.

[39] D. Wei, Diffusion and mixing in fluid flow via the resolvent estimate, Sci. China Math. 64 (2021), no. 3, 507-518.

[40] D. Wei, Z. Zhang, and W. Zhao, Linear inviscid damping and enhanced dissipation for the Kolmogorov flow, Adv. Math. 362 (2020), 106963, 103.

Michele Coti Zelati and Michele Dolce

Department of Mathematics

Imperial College, South Kensington

Campus

London SW7 2AZ

$U K$

E-mail:m.coti-zelati@imperial.ac.uk

Michele Dolce

E-mail:m.dolce@imperial.ac.uk

Yuanyuan Feng and Anna L. Mazzucato Department of Mathematics

Penn State University

University Park, PA 16802

USA

E-mail: yzf58@psu.edu

Anna L. Mazzucato

E-mail:alm24@psu.edu

Accepted: 31 August 2021 